\title{
Impact of time-to-treatment on overall survival of non-small cell lung cancer patients - an analysis of the national cancer database
}

\author{
Trisari Anggondowati ${ }^{1}$, Apar Kishor Ganti ${ }^{2}$, K. M. Monirul Islam ${ }^{3}$ \\ ${ }^{1}$ Center for Family Welfare, University of Indonesia, Kota Depok, Indonesia; ${ }^{2}$ Division of Oncology-Hematology, Department of Internal Medicine, \\ VA Nebraska-Western Iowa Health Care System and University of Nebraska Medical Center, Omaha, NE, USA; ${ }^{3}$ Institute of Public and Preventive \\ Health \& Department of Population Health Sciences, Medical College of Georgia, Augusta University, Augusta, GA, USA \\ Contributions: (I) Conception and design: All authors; (II) Administrative support: All authors; (III) Provision of study materials or patients: AK Ganti, \\ KMM Islam; (IV) Collection and assembly of data: None; (V) Data analysis and interpretation: All authors; (VI) Manuscript writing: All authors; (VII) \\ Final approval of manuscript: All authors. \\ Correspondence to: K. M. Monirul Islam. Associate Professor of Epidemiology, Institute of Public and Preventive Health and Department of \\ Population Health Sciences, Medical College of Georgia, Augusta University, 1120 15th Street, CJ 2326, Augusta, GA 30912, USA. \\ Email: kislam@augusta.edu.
}

\begin{abstract}
Background: The association between time-to-treatment and outcomes for lung cancer has not been conclusively established. In this study, we evaluated the effect of time-to-treatment on the overall 5-year survival of patients with non-small cell lung cancer (NSCLC) with cancer stage at diagnosis.

Methods: We analyzed data in the National Cancer Data Base for adult patients newly diagnosed with NSCLC in 2003-2011 (N=693,554). Extended Cox regression with counting process was used to model the effect of time-to-treatment on survival, adjusted for demographic and clinical factors. Multivariable analyses were performed separately for the groups with different stages at diagnosis. Time-to-treatment was defined as the interval between diagnosis and treatment initiation, with the categories of (I) 0 day, (II) 1 day- 4 weeks, (III) 4.1-6.0 weeks, and (IV) >6 weeks (the 1 day-4 weeks group was considered the reference group).

Results: Compared to treatment initiated between 1 day and 4 weeks after diagnosis, time-to-treatment at 4.1-6.0 weeks was associated with a lower risk of death for patients with early-stage cancer [adjusted HR (aHR), 0.84 (95\% CI, 0.82-0.85)], with locally advanced cancer [aHR, 0.82 (95\% CI, 0.80-0.83)], and with metastatic cancer [aHR, 0.75 (95\% CI, 0.74-0.76)]. Similarly, a lower risk of death was associated with timeto-treatment longer than 6 weeks for patients with any cancer stage at diagnosis. However, a subset analysis for early-stage patients who received surgery only showed that extended time-to-surgery was associated a higher risk of death [aHR $\mathrm{aH}_{4-6.0 \text { weeks, }} 1.06$ (95\% CI, 1.03-1.09); $\mathrm{aHR}_{>6 \text { weeks }} 1.17$ (95\% CI, 1.14-1.20)].

Conclusions: The findings show that, although time-to-treatment should not be compromised, it is imperative to ensure that patients receive optimal pre-treatment assessments rather than rushing the treatment. Future research should focus on examining clinical characteristics to determine an optimal timeto-treatment to achieve the best possible survival for NSCLC patients.
\end{abstract}

Keywords: Non-small cell lung cancer (NSCLC); time-to-treatment; waiting time; survival

Submitted Dec 16, 2019. Accepted for publication Jun 12, 2020.

doi: $10.21037 /$ tlcr-19-675

View this article at: http://dx.doi.org/10.21037/tlcr-19-675

\section{Introduction}

Lung cancer is the leading cause of cancer deaths in the US and worldwide (1). The 1- and 5-year overall survival (OS) rates for lung cancer patients ( $47 \%$ and $18 \%$, respectively) are lower than those of other common cancers (2). Therefore, it is appropriate to conduct research to improve understanding of factors that affect the survival of lung 
cancer patients.

In general, timely care positively affects survival of patients. Timeliness of care is defined as the system's capacity to provide care quickly after a need is recognized (3). Delayed initiation of treatment could increase psychological distress and affect the prognosis for cancer patients (4,5). A large population-based study in the US found that $36.7 \%$ of lung cancer patients experienced treatment delays (diagnosisto-treatment interval of $>35$ days) (6). Age, race, stage at diagnosis, comorbidity, and type of hospital are also associated with treatment delays $(7,8)$.

Despite the effect of time-to-treatment on outcomes for patients, previous studies of lung cancer have been inconclusive. A study of non-small cell lung cancer (NSCLC) patients aged 66 years old and older showed an adverse effect of prolonged time-to-treatment on survival (8). An adverse effect of extended time-totreatment was also found in two other studies of earlystage NSCLC patients who had surgery $(6,9)$. In contrast, other studies found that longer time-to-treatment was associated with improved survival (10-12). This association, often referred as the 'waiting time paradox,' has also been observed for colorectal and endometrial cancers (13). A possible reason for this paradox is that patients with more severe conditions tend to receive treatment quickly, but, despite the immediate treatment, their severe condition still leads to poor outcomes. The inconsistent results of studies on time-to-treatment might also be related to variations in the definition and cut-off points used as recommendations on time-to-treatment of NSCLC (14-16). For instance, in the US, a proposed recommendation of treatment initiation for NSCLC other than metastatic cancer is within 6 weeks of diagnosis (14), in the United Kingdom, the general recommendation is within 4 weeks after diagnosis (15). In the Netherlands, treatment is recommended to start within 35 calendar days of the patient's first visit to a pulmonologist (17).

Since timeliness of care is modifiable, evidence pertaining to its effect on survival is of particular importance (18). The objective of the present study was to examine the effect of time-to-treatment on the OS of NSCLC patients. We hypothesized that timeliness of care positively affect the survival of lung cancer patients. We utilized a national hospital-based dataset that provided, relative to previous studies, a more representative sample of NSCLC patients in the US. We present the following article in accordance with the STROBE Reporting Checklist (available at http:// dx.doi.org/10.21037/tlcr-19-675).

\section{Methods}

We analyzed the National Cancer Data Base (NCDB) for the period 2003-2011. The NCDB is a national, hospitalbased oncology dataset, collected from more than 1,500 facilities accredited by the Commission on Cancer (CoC). The database, jointly sponsored by the American College of Surgeons and the American Cancer Society, is estimated to represent $70 \%$ of new cancer diagnoses in the US $(19,20)$. The NCDB records information about patient demographics, clinical characteristics, treatment, and outcomes. The present study used de-identified data, and thus was exempt from review by the Institutional Review Board. The study applied a retrospective cohort design.

\section{Study population}

Patients aged 18 years and older who were diagnosed with NSCLC from 2003 to 2011 and underwent any surgery, chemotherapy, or radiation at the reporting facilities as their first-course treatment were considered eligible for this study.

\section{Outcome and predictors}

The primary outcome was 5-year OS, defined as the time from the date of diagnosis to the date of death, or the last contact if the patient was still alive or lost to follow-up (the time was censored). The primary predictor was timeto-treatment, defined as the period between diagnosis and initiation of any first-course treatment. Time-to-treatment was categorized based on the commonly recommended time-to-treatment and the proposed recommendation in the US (within the first 4 weeks and 6 weeks after diagnosis, respectively) (14). The categories of time-totreatment were: (I) 0 day, (II) 1 day to 4 weeks, (III) $4.1-$ 6.0 weeks, and (IV) $>6$ weeks, with 1 day to 4 weeks as the reference group. We distinguished 0 day from the 1 day to 4 weeks on the assumption that patients who received treatment on the same day of diagnosis might harbor specific conditions different from other patients (e.g., have less severe symptoms and were thus eligible for prompt tumor removal or require an emergent procedure). The covariates adjusted in the analysis were age at diagnosis, sex, race, urban/rural status, distance to the reporting hospital, primary payer, facility type, stage at diagnosis, histology, treatment type, and Charlson-Deyo comorbidity score because those variables can affect both decision on timing of 
treatment and patient survival. Detailed information about how variables in the NCDB are defined by the American College of Surgeons is provided elsewhere (19). The NCDB contains data from the participating hospital cancer registries.

\section{Statistical analysis}

Descriptive statistics were presented as proportions for the categorical variables, and medians with inter-quartile range (IQR) for continuous variables. In the bivariate analyses, a chi-square test was used to assess the association between each of the patient characteristics and time-to-treatment. The association between each predictor and OS was examined with a Cox proportional hazards $(\mathrm{PH})$ model.

Prior to the analysis, we examined the $\mathrm{PH}$ assumption to determine whether the hazard ratio (HR) for any pair of levels of predictors other than time-to-treatment was constant over time. Using a graphical approach and Schoenfeld's test, we identified non-proportionality of the HRs for several predictors, including time-to-treatment, suggesting that the hazard rates were not constant over time. We handled this non-proportionality of HRs differently for the time-to-treatment variable and other predictors. We considered the time-to-treatment variable as having an inherent time-dependent nature. The risk of death for the patients differed before and after receiving treatment. Prior to starting treatment, patients had a relatively similar risk to those who did not receive treatment. The risk changed after they started or completed treatment. To take into account this time-dependent risk, we applied the counting process method (21). By this approach, each case was handled as two observations: one from the time of diagnosis to receiving treatment, and one from starting treatment to either death or loss to follow up. Other predictors that did not satisfy the $\mathrm{PH}$ assumption were handled through Stratified Cox regressions (21). The counting process and stratified Cox regression were fitted in the final analyses.

Multivariable analyses were performed separately for the groups with different stages at diagnosis, which were divided into early-stage (stage I and II), locally advanced (stage III), and metastatic disease (stage IV). Subset analyses were performed for patients considered as having a relatively good prognosis (early-stage patients who received surgery only). For this purpose, patients who died within the first month after diagnosis were excluded since they were likely to have more severe clinical conditions than other patients. The Kaplan-Meier estimator was used to produce survival estimates (22). The significance level for the analysis was set at a $\mathrm{P}$ value $<0.05$. All statistical analyses were performed with the statistical software package SAS, version 9.4 (SAS Institute Inc., Cary, NC). Observations with missing variables of interest were excluded.

\section{Results}

From 2003 to 2011, the NCDB recorded 1,010,492 patients diagnosed with NSCLC. Of these, 259,425 cases who were not eligible due to, for example, not receiving treatment in the reporting facilities or having unknown treatment status, were excluded. Of 751,067 eligible cases, 59,603 $(7.9 \%)$ were excluded largely due to missing information on stage at diagnosis and time-to-treatment (Figure 1). In total, 691,464 patients were included in the analysis. There were no substantial differences between the excluded and analyzed cases, except for histology and comorbidity score. The cases included in the analysis had a higher proportion of adenocarcinoma (46.4\%) and comorbidity scores of either 1 or $2(39.3 \%)$ compared to the excluded cases $(37.3 \%$ and $33.8 \%$, respectively). The median follow-up period was 15 months (IQR $=30$ ).

\section{Descriptive analysis of time-to-treatment and characteristics of patients}

Overall, $42.6 \%$ of patients started treatment more than 4 weeks after diagnosis. The median time-to-treatment was different between patients with metastatic cancer [18 days, (IQR, 11-36)] and those with less advanced stages. The medians for patients with early-stage and locally advanced disease was 28 days (IQR, 2-51) and 27 days (IQR, 13-46), respectively.

Demographic and clinical characteristics of patients are presented in Table 1. Time-to-treatment was significantly associated with all patient demographic and clinical characteristics analyzed (see the note of Table 1). The proportion of patients receiving treatment within the first 4 weeks of diagnosis was higher for those who were younger than 65 years, with high income, had private insurance, diagnosed with early-stage disease, or had surgery. Patients with Medicare (mostly age $\geq 65$ years) tended to start treatment more than 6 weeks after diagnosis. The median OS time was 54 months (IQR =95) for early-stage, 15 months (IQR =29.2) for a locally advanced stage, and 6.4 months $(\mathrm{IQR}=11.3)$ for patients with metastatic cancer. 


\begin{tabular}{|c|c|}
\hline $\begin{array}{l}\text { NSCLC diagnosed from } 2003 \text { to } 2011 \\
\qquad(\mathrm{~N}=1,010,492)\end{array}$ & Excluded cases \\
\hline$\downarrow$ & Diagnosed at the reporting facility, but treatment decisions were made elsewhere $(\mathrm{N}=83,183)$ \\
\hline \multicolumn{2}{|l|}{$\mathrm{N}=927,309$} \\
\hline$\downarrow$ & $\begin{array}{l}\text { - Did not receive first-course treatment at the reporting facility }(\mathrm{N}=158,245) \\
\text { - Treatment status was unknown }(\mathrm{N}=15,605) \\
\text { - Received only hormone or immunotherapy }(\mathrm{N}=2,392)\end{array}$ \\
\hline \multicolumn{2}{|l|}{ Eligible cases $(\mathrm{N}=751,067)$} \\
\hline$\downarrow$ & $\begin{array}{l}\text { - Missing information on stage at diagnosis }(\mathrm{N}=40,925) \\
\text { - Stage at diagnosis was either } 0 \text { or occult }(\mathrm{N}=1,355)\end{array}$ \\
\hline \multicolumn{2}{|l|}{$\mathrm{N}=708,787$} \\
\hline Analyzed $(\mathrm{N}=691,464)$ & \\
\hline
\end{tabular}

Figure 1 Selection of the study population.

\section{The effect of time-to-treatment on OS}

The multivariable analyses showed different effects of timeto-treatment on OS between patients with an early- and advanced-stage diseases.

\section{Early-stage disease}

The multivariable analyses (Table 2) showed a lower risk of death for patients who received treatment on the day of diagnosis, compared to those who initiated treatment between 1 day and 4 weeks after diagnosis [adjusted HR $(\mathrm{aHR})=0.84(95 \%$ CI, 0.82-0.85)]. Similarly, a lower risk of death was associated with time-to-treatment longer than 4 weeks [aHR, 0.93 (95\% CI, 0.91-0.95)] and longer than 6 weeks after diagnosis [aHR, 0.92 (95\% CI, 0.91-0.94)] (Table 2). However, a subset analysis for patients who received surgery only, showed that surgery between 4 and 6 weeks was associated with a $6 \%$ increased risk of death [aHR, 1.06 (95\% CI, 1.03-1.09)]. A higher risk of death (17\%) was evident for early-stage patients who received surgery more than 6 weeks after diagnosis [aHR, 1.17 (95\% CI, 1.14-1.20)] (Table 3).

\section{Locally-advanced stage}

Compared to treatment initiated 1 day to 4 weeks after diagnosis, either a shorter or longer time-to-treatment was associated with a lower risk of death $\left[\mathrm{aHR}_{0}\right.$ day $0.73(95 \%$ CI, 0.71-0.74]; aHR $4.1-6.0$ weeks 0.82 (95\% CI, 0.80-0.83];
$\left.\mathrm{aHR}_{>6 \text { weeks }} 0.71(95 \% \mathrm{CI}, 0.70-0.72)\right]$.

\section{Metastatic cancer}

Similar to findings for patients with locally advanced disease, there was a lower risk of death for patients with metastatic cancer who received treatment at the same day of diagnosis or after 4 weeks $\left[\mathrm{aHR}_{0 \text { day }} 0.75\right.$ (95\% CI, $0.73-$ $0.76) ; \mathrm{aHR}_{4.1-6.0 \text { weeks }} 0.75$ (95\% CI, 0.73-0.76); $\mathrm{aHR}_{>6 \text { weeks }}$ 0.58 (95\% CI, 0.57-0.59)].

\section{Discussion}

The Institute of Medicine has established timeliness of care as a dimension of healthcare quality $(23,24)$. However, delay in receiving treatment remains a problem for some cancer patients $(7,8)$. Although the present study did not prove the hypothesis of adverse effects of extended time-to-treatment on the OS of NSCLC patients, extended time-to-treatment was an independent predictor of mortality for early-stage patients who received surgery.

The present findings indicate a complex association between time-to-treatment and survival. Contrary to our hypothesis, time-to-treatment longer than four weeks was associated with a lower risk of death for patients of all cancer stages. For early-stage patients, however, the difference in risk of death by time-to-treatment is subtle compared to that for patients with locally advanced or 
Table 1 Patients' socio-demographic and clinical characteristics by time-to-treatment ${ }^{\mathrm{a}}$

\begin{tabular}{|c|c|c|c|c|c|}
\hline Characteristics & 0 days, $\mathrm{n}(\%)$ & 1 day-4 weeks, n (\%) & 4.1-6 weeks, n (\%) & $>6$ weeks, $\mathrm{n}(\%)$ & Total, n (\%) \\
\hline Male & 49,251 (48.9) & $164,691(55.7)$ & $62,251(54.1)$ & $94,263(52.4)$ & $370,456(53.6)$ \\
\hline Female & $51,536(51.1)$ & $131,060(44.3)$ & $52,841(45.9)$ & $85,571(47.6)$ & $321,008(46.4)$ \\
\hline \multicolumn{6}{|l|}{ Age at diagnosis, years } \\
\hline $65-74$ & $37,243(37.0)$ & $97,484(33.0)$ & 40,641 (35.3) & $63,675(35.4)$ & 239,043 (34.6) \\
\hline $75+$ & $24,189(24.0)$ & $72,642(24.6)$ & $33,380(29.0)$ & $58,426(32.5)$ & $188,637(27.3)$ \\
\hline \multicolumn{6}{|l|}{ Racial group } \\
\hline White & $89,131(88.4)$ & $255,578(86.4)$ & $100,561(87.4)$ & $151,589(84.3)$ & $596,859(86.3)$ \\
\hline Unknown & $849(0.8)$ & $2,215(0.8)$ & $903(0.8)$ & $1,380(0.8)$ & $5,347(0.8)$ \\
\hline \multicolumn{6}{|l|}{ Urban/rural } \\
\hline Metro & $80,011(79.4)$ & $227,819(77.0)$ & $88,734(77.1)$ & $141,738(78.8)$ & $538,302(77.9)$ \\
\hline Urban & $14,332(14.2)$ & $47,290(16.0)$ & $18,613(16.2)$ & $26,752(14.9)$ & $106,987(15.5)$ \\
\hline Rural & $1,867(1.9)$ & 6,952 (2.4) & $2,560(2.2)$ & 3,427 (1.9) & $14,806(2.1)$ \\
\hline Unknown & $4,577(4.5)$ & $13,690(4.6)$ & $5,185(4.5)$ & $7,917(4.4)$ & $31,369(4.5)$ \\
\hline \multicolumn{6}{|l|}{ Distance to hospital, miles } \\
\hline$\leq 10$ & 48,695 (48.3) & $155,000(52.4)$ & $59,042(51.3)$ & $93,773(52.2)$ & $35,651(51.6)$ \\
\hline Not insured & $2,127(2.1)$ & $11,524(3.9)$ & $2,843(2.5)$ & 4,642 (2.6) & $21,136(3.1)$ \\
\hline Private insurance & $34,327(34.1)$ & $96,985(32.8)$ & $34,205(29.7)$ & $45,406(25.3)$ & $210,923(30.5)$ \\
\hline Medicaid & $4,318(4.3)$ & $17,905(6.1)$ & $5,717(5.0)$ & $10,657(5.9)$ & $38,597(5.6)$ \\
\hline Medicare & $56,907(56.5)$ & $159,124(53.8)$ & $68,263(59.3)$ & $111,653(62.1)$ & $395,947(57.3)$ \\
\hline Other government insurance & $1,025(1.0)$ & $3,539(1.2)$ & $1,464(1.3)$ & $2,871(1.6)$ & $8,899(1.3)$ \\
\hline Unknown & $2,083(2.1)$ & $6,674(2.3)$ & $2,600(2.3)$ & $4,605(2.6)$ & $15,962(2.3)$ \\
\hline \multicolumn{6}{|l|}{ Facility type } \\
\hline CCP & $8,828(8.8)$ & $34,131(11.5)$ & $12,951(11.3)$ & $18,899(10.5)$ & 74,809 (10.8) \\
\hline СCCP & $54,302(53.9)$ & $174,009(58.8)$ & 64,497 (56.0) & 93,999 (52.3) & $386,807(55.9)$ \\
\hline Academic/research program & $37,357(37.1)$ & $87,141(29.5)$ & $37,510(32.6)$ & $66,760(37.1)$ & $228,768(33.1)$ \\
\hline Other & $300(0.3)$ & $470(0.2)$ & $134(0.1)$ & $176(0.1)$ & $1,080(0.2)$ \\
\hline
\end{tabular}

Table 1 (continued) 
Table 1 (continued)

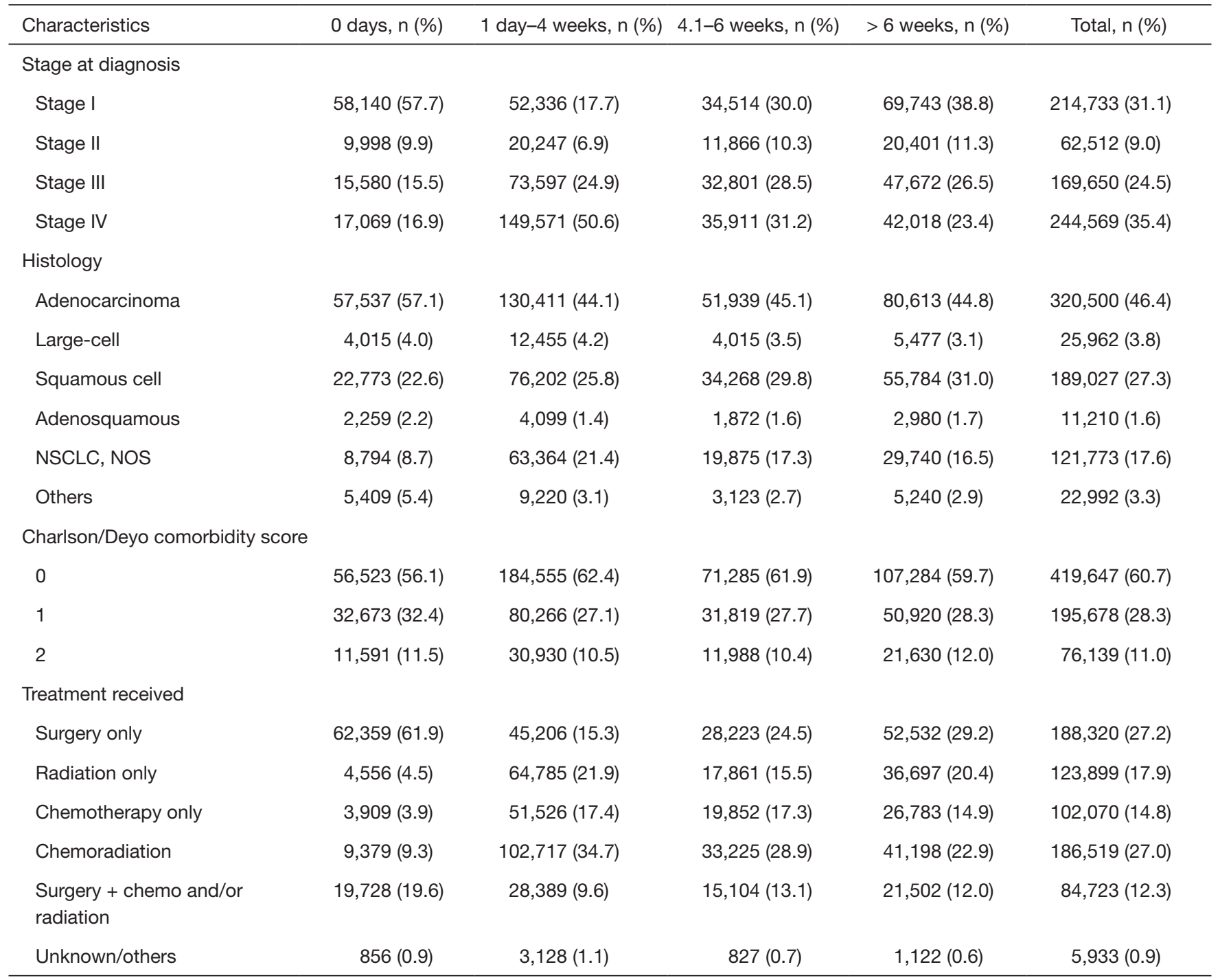

${ }^{a}$, the chi-square test for all comparisons resulted in a $\mathrm{P}$ value $<0.001$. CCP, Community Cancer Program; CCCP, comprehensive community cancer program; NSCLC, non-small cell lung cancer; NOS, not otherwise specified; IQR, inter-quartile range.

Table 2 Adjusted hazard ratios [aHR (95\% CI)] for 5-year mortality associated with time-to-treatment*

\begin{tabular}{lccc}
\hline Time-to-treatment & Early-stage $(\mathrm{N}=277,245)$ & Locally advanced disease $(\mathrm{N}=169,650)$ & Metastatic disease $(\mathrm{N}=244,569)$ \\
\hline 0 day & $0.84(0.82-0.85)$ & $0.73(0.71-0.74)$ & $0.75(0.74-0.76)$ \\
1 day to 4 weeks & Ref. & Ref. & Ref. \\
$4.1-6.0$ weeks & $0.93(0.91-0.95)$ & $0.82(0.80-0.83)$ & $0.75(0.74-0.76)$ \\
$>6$ weeks & $0.92(0.91-0.94)$ & $0.71(0.70-0.72)$ & $0.58(0.57-0.59)$ \\
\hline
\end{tabular}

*, model was adjusted for age, sex, race, urban/rural, distance to hospital, facility type, primary payer, Charlson/Deyo comorbidity score, histologic type, and treatment modalities. 
Table 3 Adjusted hazard ratios [aHR (95\% CI)] for 5-year mortality, associated with time-to-treatment for early-stage patients who received surgery only*

\begin{tabular}{lc}
\hline Time-to-treatment & aHR $(95 \% \mathrm{Cl})$ \\
\hline 0 day & $0.91(0.89-0.93)$ \\
1 day to 4 weeks & Ref. \\
$4.1-6.0$ weeks & $1.06(1.03-1.09)$ \\
$>6$ weeks & $1.17(1.14-1.20)$ \\
\hline
\end{tabular}

*, model was adjusted for age, sex, race, urban/rural, distance to hospital, facility type, primary payer, Charlson/Deyo comorbidity score, and histologic type. Excluding patients who died within 1 month after diagnosis.

metastatic cancers. However, a subset analysis for earlystage patients who received surgery alone and were considered as having a better prognosis than the other groups, showed an association in the opposite direction. Compared to treatment initiated within 1 day to 4 weeks, a longer time-to-treatment for these patients was associated with a decreased 5-year survival. Although the increased risk was modest for those who received treatment within 1 day to 4 weeks, the risk was amplified for patients who were treated more than 6 weeks after diagnosis, providing evidence of the effect of time-to-treatment.

Our results were generally inconsistent with previous findings. A study with US veterans showed an increased risk of death associated with greater timely care [aHR, 1.6 (95\% CI, 1.3-1.9)], independent of the type of treatment (25). Another study of Medicare beneficiaries found varying results, of which an improved OS was associated with a diagnosis-to-treatment interval of $\leq 35$ days for localized disease [aHR, 0.86 (95\% CI, $0.80-0.91)]$ and for patients with distant disease who survived $\geq 1$ year [aHR, 0.86 (95\% CI, 0.74-0.99)], but was associated with a lower OS for patients who survived $<1$ year (8). Nevertheless, our findings for early-stage patients who received surgery were consistent with results reported for two previous studies. A study using NCDB data for patients with stage I NSCLC, but with a different analytical approach, found that each week of delay of surgery increased the hazard of death by $0.4 \%$ [aHR, 1.004 (95\% CI, 1.002-1.007)] (9). Another study on delay in surgery for patients diagnosed at a community center suggested a similar association that was not statistically significant [aHR, 1.04 (95\% CI, 1.00-1.09)] (6).

Similar to our overall analysis, counter-intuitive results that extended time-to-treatment is associated with longer survival have been reported for studies, not limited to lung cancer, in the US and other countries $(13,26)$. This phenomenon is commonly referred as the 'waiting time paradox' (27). The premise behind this paradox is that the association between time-to-treatment and OS is likely affected by the disease severity at presentation. Patients who are treated early may have severe symptoms and inherently have a worse prognosis compared to patients treated with longer time-to-treatment. Another possible reason for the finding is that, for patients with shorter time-to-treatment, the treatment plan might involve a less comprehensive evaluation that puts patients at risk for a worse prognosis.

Our stratification analysis by disease stage failed to distinguish the severity level of patients that affects both time-to-treatment and survival, suggesting a mix of patients' risk within the same stage. For early-stage patients who received surgery, however, the patient population was likely more homogenous with respect to their clinical conditions. Therefore, the subset analysis for this population showed the adverse effect of extended time-to-treatment.

The adverse effect of extended time-to-treatment on OS can be explained by disease progression. A prolonged waiting period might cause some patients to become ineligible for curative-intent therapy, thus reducing their chance of cure. This premise has been demonstrated by studies of radiotherapy $(4,28)$. A prospective study of 29 lung cancer patients waiting for radical radiotherapy showed that $21 \%$ of potentially curable patients became incurable, and the cross-sectional tumor size increased more than three-fold times during the waiting period (4).

The strengths of the present study mainly relate to the wide coverage of the database and the large sample size. The sample size allowed us to perform stratification analyses, without being underpowered. Furthermore, the database enabled us to account for various potential confounders. In a study of colorectal cancer, control for more comprehensive confounders led to a different interpretation of the effect of time-to-treatment on OS (26). Our analysis included patients with various primary cancers, who are commonly excluded from analyses using cancer registry data (29). Our inclusion might lead to a higher risk of death in our study cohort. However, a subset analysis of patients with a single primary cancer yielded similar results (data not shown), as was found in other studies $(29,30)$.

Our results have limitations. The NCDB contains data of patients whose diagnosis was made and/or received treatment in the participating hospitals, i.e., facilities 
accredited by the CoC. Although the NCDB covers about $70 \%$ of newly diagnosed cancer cases in the US $(19,20)$, the data has potential selection bias because the choice of hospital may be associated with certain patients factors. If patients who are treated in the NCDB participating hospitals have higher severity than patients from the rest of the hospitals across US, then the bias is likely towards the null value. The CoC-approved hospitals included in the NCDB are typically larger, located in urban locations, and provide a higher degree of oncology-related specialization, compared to nonCoC-approved hospitals (31). Thus, the data might not be representative of all hospitals in the US. The influence of unmeasured confounders, particularly those that may affect the decision to expedite or postpone treatment (e.g., tumor aggressiveness, performance status), was likely the reason for the counter-intuitive findings for locally advanced and metastatic NSCLC.

Our results did not lead us to conclude that longer timeto-treatment leads to better survival. Instead, we believe that longer time-to-treatment does not have detrimental effects for patients, especially those with more advanced disease stages. Therefore, comprehensive examinations and preparation will benefit patients more, rather than rushing the treatment. However, for patients with operable cancers, it is important not to delay treatment. Future research should focus on identifying, for patients, clinical characteristics that could predict the tolerable time-totreatment to achieve better outcomes, which would be beneficial for patients and provide a basis for improving clinical standards. Such research would also be relevant, given the fact that guidelines vary for timing of treatment initiation of NSCLC.

\section{Conclusions}

The study supports the idea that allocating time for optimal pre-treatment assessments, although leading to a longer time-to-treatment interval, is likely to benefit survival of patients. However, it seems prudent for treatment of patients with resectable, early-stage lung cancer to start within 4 weeks of diagnosis. This relates to the expected increased identification of early-stage patients following improvements in screening programs and recent recommendations from the US Preventive Services Task Force. Despite the various factors that lead to treatment delays, attempts should be made to decrease delays caused by system-based factors.

\section{Acknowledgments}

The authors would like to thank Ann Fetrick, $\mathrm{PhD}$, and June Ryan, MPA, for providing editorial assistance. The data used in the study are derived from a de-identified NCDB file. The American College of Surgeons and the Commission on Cancer have not verified and are not responsible for the analytic or statistical methodology employed, or the conclusions drawn from these data by the investigators.

Funding: This work was supported in part by the PatientCentered Outcomes Research Institute (PCORI) [Contract \#CE-12-11-4351] and the Fred \& Pamela Buffett Cancer Center Support Grant [P30CA036727]. The ideas and opinions expressed herein are those of the authors and do not necessarily represent the official views of PCORI.

\section{Footnote}

Reporting Checklist: The authors have completed the STROBE Reporting Checklist. Available at http://dx.doi. org/10.21037/tlcr-19-675

Conflicts of Interest: All authors have completed the ICMJE uniform disclosure form (available at http://dx.doi. org/10.21037/tlcr-19-675). AKG reports non-financial support from Takeda, grants from Apexigen, grants and personal fees from AstraZeneca, personal fees from Roche, grants from Novartis, grants from Merck, outside the submitted work. The other authors have no conflicts of interest to declare.

Etbical Statement: The authors are accountable for all aspects of the work in ensuring that questions related to the accuracy or integrity of any part of the work are appropriately investigated and resolved. The present study used de-identified data, and thus was exempt from review by the Institutional Review Board.

Open Access Statement: This is an Open Access article distributed in accordance with the Creative Commons Attribution-NonCommercial-NoDerivs 4.0 International License (CC BY-NC-ND 4.0), which permits the noncommercial replication and distribution of the article with the strict proviso that no changes or edits are made and the original work is properly cited (including links to both the formal publication through the relevant DOI and the license). See: https://creativecommons.org/licenses/by-nc-nd/4.0/. 


\section{References}

1. Ferlay J, Soerjomataram I, Ervik M, et al. GLOBOCAN 2012: Estimated Cancer Incidence, Mortality and Prevalence Worldwide in 2012 v1.0. IARC CancerBase No. 11.

2. Howlader N, Noone A, Krapcho M, et al. SEER Cancer Statistics Review (CSR) 1975-2014. Published April 2017. Updated April 2017. Accessed Sep/1, 2017. Available online: https://seer.cancer.gov/csr/1975_2014/

3. Agency for Healthcare Research and Quality. National healthcare disparities report 2011. Rockville, MD, 2012; Available online: http://archive.ahrq.gov/research/ findings/nhqrdr/nhdr11/index.html

4. O'Rourke N, Edwards R. Lung cancer treatment waiting times and tumour growth. Clin Oncol (R Coll Radiol) 2000;12:141-4.

5. Paul C, Carey M, Anderson A, et al. Cancer patients' concerns regarding access to cancer care: Perceived impact of waiting times along the diagnosis and treatment journey. Eur J Cancer Care (Engl) 2012;21:321-9.

6. Kanarek NF, Hooker CM, Mathieu L, et al. Survival after community diagnosis of early-stage non-small cell lung cancer. Am J Med 2014;127:443-9.

7. Bilimoria KY, Ko CY, Tomlinson JS, et al. Wait times for cancer surgery in the United States: Trends and predictors of delays. Ann Surg 2011;253:779-85.

8. Gomez DR, Liao KP, Swisher SG, et al. Time to treatment as a quality metric in lung cancer: Staging studies, time to treatment, and patient survival. Radiother Oncol 2015;115:257-63.

9. Samson P, Patel A, Garrett T, et al. Effects of delayed surgical resection on short-term and long-term outcomes in clinical stage I non-small cell lung cancer. Ann Thorac Surg 2015;99:1906-12.

10. Myrdal G, Lambe M, Hillerdal G, et al. Effect of delays on prognosis in patients with non-small cell lung cancer. Thorax 2004;59:45-9.

11. Nadpara P, Madhavan SS, Tworek C. Guidelineconcordant timely lung cancer care and prognosis among elderly patients in the United States: A population-based study. Cancer Epidemiol 2015;39:1136-44.

12. Diaconescu R, Lafond C, Whittom R. Treatment delays in non-small cell lung cancer and their prognostic implications. J Thorac Oncol 2011;6:1254-9.

13. Crawford SC, Davis JA, Siddiqui NA, et al. The waiting time paradox: Population based retrospective study of treatment delay and survival of women with endometrial cancer in scotland. BMJ 2002;325:196.

14. Reifel JL. Lung cancer. In: Asch SM, Kerr EA, Hamilton $\mathrm{EG}$, et al. editors. Quality of care for oncologic conditions and HIV: A review of the literature and quality indicators. Santa Monica, CA, USA: RAND, 2000.

15. Department of Health, National Health Services (NHS) UK. The NHS cancer plan: A plan for investment, A plan for reform. 2000.

16. Vinas F, Ben Hassen I, Jabot L, et al. Delays for diagnosis and treatment of lung cancers: A systematic review. Clin Respir J 2016;10:267-71.

17. Hermens RP, Ouwens MM, Vonk-Okhuijsen SY, et al. Development of quality indicators for diagnosis and treatment of patients with non-small cell lung cancer: A first step toward implementing a multidisciplinary, evidence-based guideline. Lung Cancer 2006;54:117-24.

18. Moody A, Muers M, Forman D. Delays in managing lung cancer. Thorax 2004;59:1-3.

19. American College of Surgeons Commission on Cancer. National cancer data base PUF. Updated 2015. Accessed May/1, 2015. Available online: http://ncdbpuf.facs.org/

20. Lerro CC, Robbins AS, Phillips JL, et al. Comparison of cases captured in the national cancer data base with those in population-based central cancer registries. Ann Surg Oncol 2013;20:1759-65.

21. Kleinbaum D, Klein M. Survival analysis: A self-learning text. 3rd edition. New York: Springer, 2012.

22. Allison PD. Survival analysis using SAS: A practical guide. 2nd edition. SAS Institute Inc., Cary, NC, 2010.

23. Institute of Medicine (IOM). Access to health care in America. Washington, DC: National Academic Press, 1993.

24. Institute of Medicine (IOM). Transforming health care scheduling and access: Getting to now. Washington, DC: National Academies Press, 2015.

25. Gould MK, Ghaus SJ, Olsson JK, et al. Timeliness of care in veterans with non-small cell lung cancer. Chest 2008;133:1167-73.

26. Murchie P, Raja EA, Brewster DH, et al. Time from first presentation in primary care to treatment of symptomatic colorectal cancer: Effect on disease stage and survival. Br J Cancer 2014;111:461-9.

27. Neal RD, Tharmanathan P, France B, et al. Is increased time to diagnosis and treatment in symptomatic cancer associated with poorer outcomes? Systematic review. Br J Cancer 2015;112 Suppl 1:S92-107.

28. Everitt S, Plumridge N, Herschtal A, et al. The impact of time between staging PET/CT and definitive chemoradiation on target volumes and survival in patients 
with non-small cell lung cancer. Radiother Oncol 2013;106:288-91.

29. Rosso S, De Angelis R, Ciccolallo L, et al. Multiple tumours in survival estimates. Eur J Cancer 2009;45:1080-94.

30. Ellison LF. Measuring the effect of including multiple cancers in survival analyses using data from the Canadian
Cancer Registry. Cancer Epidemiol 2010;34:550-5.

31. Bilimoria KY, Bentrem DJ, Stewart AK, et al. Comparison of commission on cancer-approved and -non approved hospitals in the United States: Implications for studies that use the National Cancer Data Base. J Clin Oncol 2009;27:4177-81.
Cite this article as: Anggondowati T, Ganti AK, Islam KMM. Impact of time-to-treatment on overall survival of non-small cell lung cancer patients-an analysis of the national cancer database. Transl Cancer Res 2020;9(4):1202-1211. doi: 10.21037/ tlcr-19-675 